\title{
Beija-flores (Aves, Trochilidae) e seus recursos florais em uma área urbana do Sul do Brasil
}

\author{
Luciana Baza Mendonça \& Luiz dos Anjos
}

Laboratório de Ornitologia e Bioacústica, Departamento de Biologia Vegetal e Animal, Universidade Estadual de Londrina. Caixa Postal 6001, 86051-970 Londrina, Brasil. E-mail: lu_baza@yahoo.com.br

\begin{abstract}
Hummingbirds (Aves, Trochilidae) and their flowers in an urban area of southern Brazil. The main purpose of this study was to identify the assemblage of flowers used by hummingbirds in an urban area of southern Brazil, as well as describe the patterns of resource partitioning among the hummingbird species. Twenty two plant species were recorded, which flowers are visited by ten hummingbird species. Most of these plants are exotics and regarded as non-ornithophilous. The hummingbirds displayed different combinations of morphological features (mass, bill length, wing length and relation bill/wing), which were reflected in the sets of flowers visited and the community role played by each species. In general, hummingbirds with largest niche breadths were also the most frequent ones, indicating that urban areas could be more suitable for generalist species.
\end{abstract}

KEY WORDS. bird-plant interactions, nectarivorous birds, community organization, Paraná, urbanization.

RESUMO. A assembléia de flores visitadas por beija-flores e a partilha de recursos entre estas aves foram estudadas em uma área urbana do sul do Brasil.Vinte e duas espécies de plantas, em sua maioria exóticas e/ou não-ornitófilas, receberam visitas dos beija-flores. As espécies de beija-flores registradas apresentaram diferentes combinações de características morfológicas (massa, comprimento do bico, comprimento da asa e relação bico/ asa), que refletiram no conjunto de flores exploradas e no papel comunitário desempenhado por cada espécie. De um modo geral, beija-flores com nichos tróficos mais amplos foram também os mais freqüentes na área de estudo, indicando que o ambiente urbano pode ser mais favorável às espécies generalistas. PALAVRAS-CHAVE. aves nectarívoras, interação ave-planta, nicho trófico, Paraná, urbanização.

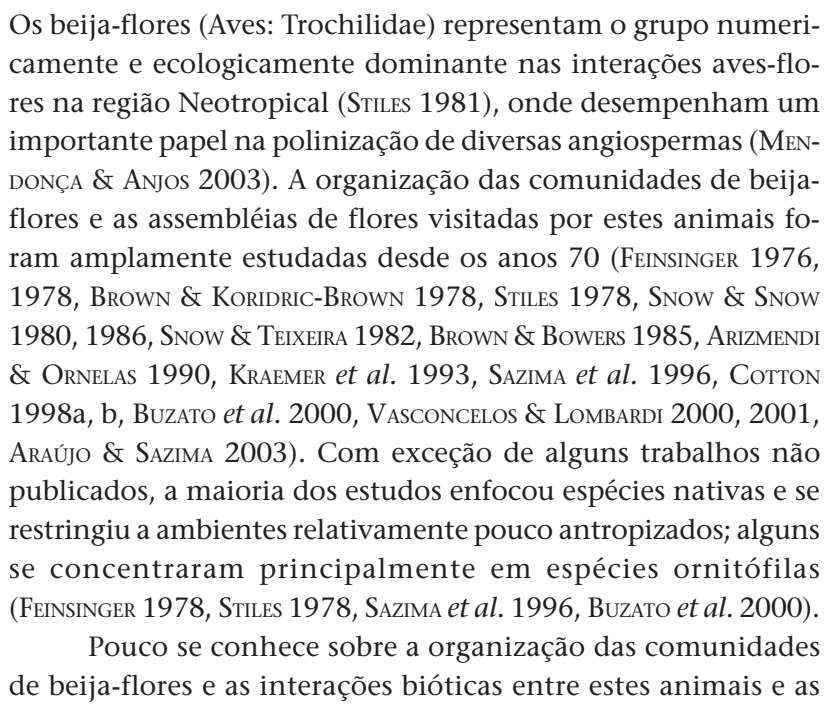

flores em ambientes urbanos, além das informações obtidas em estudos sobre a composição e distribuição espacial da avifauna (e.g. Westcotт 1980). A urbanização modifica significativamente a estrutura física e biótica do hábitat, podendo afetar diversos processos ecológicos que envolvem a fauna e a flora nestas áreas. Como resultado da intervenção antrópica, a paisagem urbana geralmente se apresenta fragmentada em um mosaico de diferentes ambientes e tanto a estrutura da vegetação quanto a sua composição florística costumam diferir daquela originalmente presente (ArgeL-De-OliveIra 1996); a paisagem urbana disponibiliza, portanto, condições e recursos distintos a serem explorados pela fauna.

Considerando os recursos florais disponíveis e a estrutura da paisagem nas áreas urbanas, o presente trabalho tem como objetivo principal investigar: (1) quais espécies compõem a assembléia de plantas exploradas pelos beija-flores em uma paisagem urbana e (2) como se dá a partição dos recursos florais entre as espécies. 


\section{MATERIAL E MÉTODOS}

\section{Área de Estudo}

O estudo foi conduzido no campus da Universidade Estadual de Londrina (UEL 230 ha; $23^{\circ} 19^{\prime} \mathrm{S}, 51^{\circ} 12^{\prime} \mathrm{W}$ ), em Londrina, Paraná. O clima da região (Classificação de Köppen) é do tipo Cfa, com queda da precipitação no inverno. A temperatura média anual é de cerca de $21^{\circ} \mathrm{C}$, variando de $27^{\circ} \mathrm{C}$ no verão a $15,5^{\circ} \mathrm{C}$ no inverno. A precipitação média anual é em torno de $1600 \mathrm{~mm}$.

Londrina está inserida na região fitogeográfica da Floresta Estacional Semidecidual, onde Aspidosperma polyneuron Müll. Arg. (peroba) se constitui numa das árvores emergentes mais características na região. No campus da UEL, a vegetação original foi quase que totalmente removida no passado e atualmente é caracterizada por algumas espécies arbóreas remanescentes, como A. polyneuron e Ficus sp. (figueiras) e espécies nativas e exóticas, sobretudo arbóreas e arbustivas, plantadas ao longo de grandes extensões de gramado. Encontra-se ainda um fragmento de floresta secundária (horto florestal) de aproximadamente 10 ha, o qual não foi incluído nas análises.

\section{Observações de Campo}

O estudo foi realizado de abril a agosto de 2000, totalizando 238 h de observação. Os beija-flores e as plantas com flores foram observados semanalmente ao longo de uma transecção com 2 km de extensão, abrangendo uma área amostral de cerca de 10 ha. As observações foram conduzidas em dois períodos do dia, com duração média de três horas por período: de manhã (iniciando uma hora após o sol nascente) e à tarde (até uma hora antes do sol poente). Adicionalmente, foram realizadas sessões não sistematizadas de observação focal, com duração variável (60-180 min) nas espécies floridas. Dias com chuva foram desconsiderados.

Para cada espécie de planta visitada, foram registrados o hábito, a morfologia floral e a(s) cor(es) predominante(s) nas flores. A fenologia de floração (presença/ausência de flores) foi acompanhada mensalmente em campo. Medidas do comprimento interno da corola, da base até a abertura (comprimento efetivo sensu Wolf et al. 1976) foram obtidas em material fresco ou flores fixadas em álcool 70\%, com o uso de paquímetro digital (precisão de $0,01 \mathrm{~mm}$ ). As síndromes de polinização foram assinaladas principalmente com base nas características morfológicas das flores (FAEGRI \& VAN DER PIJL 1979), literatura e observação de visitantes em campo. A nomenclatura e autores das plantas seguem o Missouri Botanical Garden (2004).

Os beija-flores foram observados a vista desarmada ou com o auxílio de binóculos. Ao ser observada uma visita, registrouse a espécie de beija-flor, a espécie de planta visitada, o comportamento e as interações agonísticas intra e interspecíficas.

\section{Análise dos dados}

A freqüência de ocorrência dos beija-flores foi baseada no número de dias em que uma dada espécie foi observada em relação ao número total de dias em que as observações foram conduzidas $(\mathrm{N}=45)$. A identificação e os dados morfológicos das espécies de beija-flores seguem GranTSAU (1988) e os papéis comunitários desempenhados pelas diferentes espécies foram definidos com base em Feinsinger \& Colwell (1978). Utilizou-se também a relação proposta por SNOW \& SNOW (1980) entre o comprimento do bico e o comprimento da asa, para explorar correspondência entre a estratégia de forrageamento adotada e os atributos morfológicos das espécies.

Quando do contato agressivo entre beija-flores de espécies diferentes, o beija-flor dominante foi considerado aquele que afastou o outro das flores e permaneceu próximo ao recurso floral. A posição das diferentes espécies na hierarquia de dominância foi medida como a porcentagem dos encontros interespecíficos ganhos. As correlações apresentadas no texto são r-Spearman.

A amplitude do nicho trófico de cada espécie de beija-flor foi calculada pelo índice de Shannon (KREBs 1999), com a fórmula $H^{\prime}=$ - å $\left(p_{\mathrm{i}} \ln \mathrm{p}_{\mathrm{i}}\right)$, onde $\mathrm{p}_{\mathrm{i}}$ é a proporção das visitas realizadas à planta i. A equitabilidade das visitas de cada espécie de beija-flor às plantas floridas foi calculada com a fórmula $\mathrm{J}^{\prime}=\mathrm{H}^{\prime} / \mathrm{H}^{\prime}{ }_{\text {max }}$, onde $\mathrm{H}_{\text {max }}^{\prime}$ é o logaritmo neperiano do número total de espécies de plantas visitadas pela espécie de beija-flor considerada.

A sobreposição dos nichos tróficos das espécies foi calculada com o índice de Pianka (Krebs 1999), da seguinte ma-

neira: $O_{j k}=\frac{\sum p_{i j} p_{i k}}{\sqrt{\sum p_{i j}^{2} \sum p_{j k}^{2}}}$, onde $\mathrm{p}_{\mathrm{i}}$ é a proporção das visi-

tas da espécie j ou k ao recurso i. Este índice varia de 0 (nenhuma sobreposição) a 1 (sobreposição completa). Como uma visita considerou-se cada dia em que uma dada espécie de beijaflor foi observada em uma dada espécie de planta.

\section{RESULTADOS}

\section{Assembléia de plantas visitadas}

Os beija-flores visitaram um total de 22 espécies vegetais, em sua maioria exóticas (> 60\%), distribuídas em 13 famílias e 20 gêneros (Tab. I). Das espécies registradas, 45,4\% são arbóreas e 40,9\% são arbustivas. Dez espécies $(45,4 \%)$, pertencentes a seis famílias, apresentaram flores com características relacionadas à ornitofilia. As demais (12) apresentaram características associadas a outras síndromes de polinização, sobretudo entomofilia/ melitofilia. O comprimento da corola das flores variou de 6,91 \pm 0,54 mm (Dracaena fragrans (L.) Ker-Gawl, N = 7) a 76,59 $\pm 7,44$ $\mathrm{mm}$ (Spathodea campanulata P. Beauv., $\mathrm{N}=13$ ). Seis espécies apresentaram flores durante todos os meses amostrados e florescem durante todo o ano ou a maior parte deste (L. B. Mendonça, obs. pessoal): Sanchezia nobilis Hook. f., Thunbergia grandiflora Roxb., Carica papaya L., Hibiscus rosa-sinensis L., Malvaviscus arboreus Cav. e Bouganvillea spectabilis Willd. (Tab. I). 


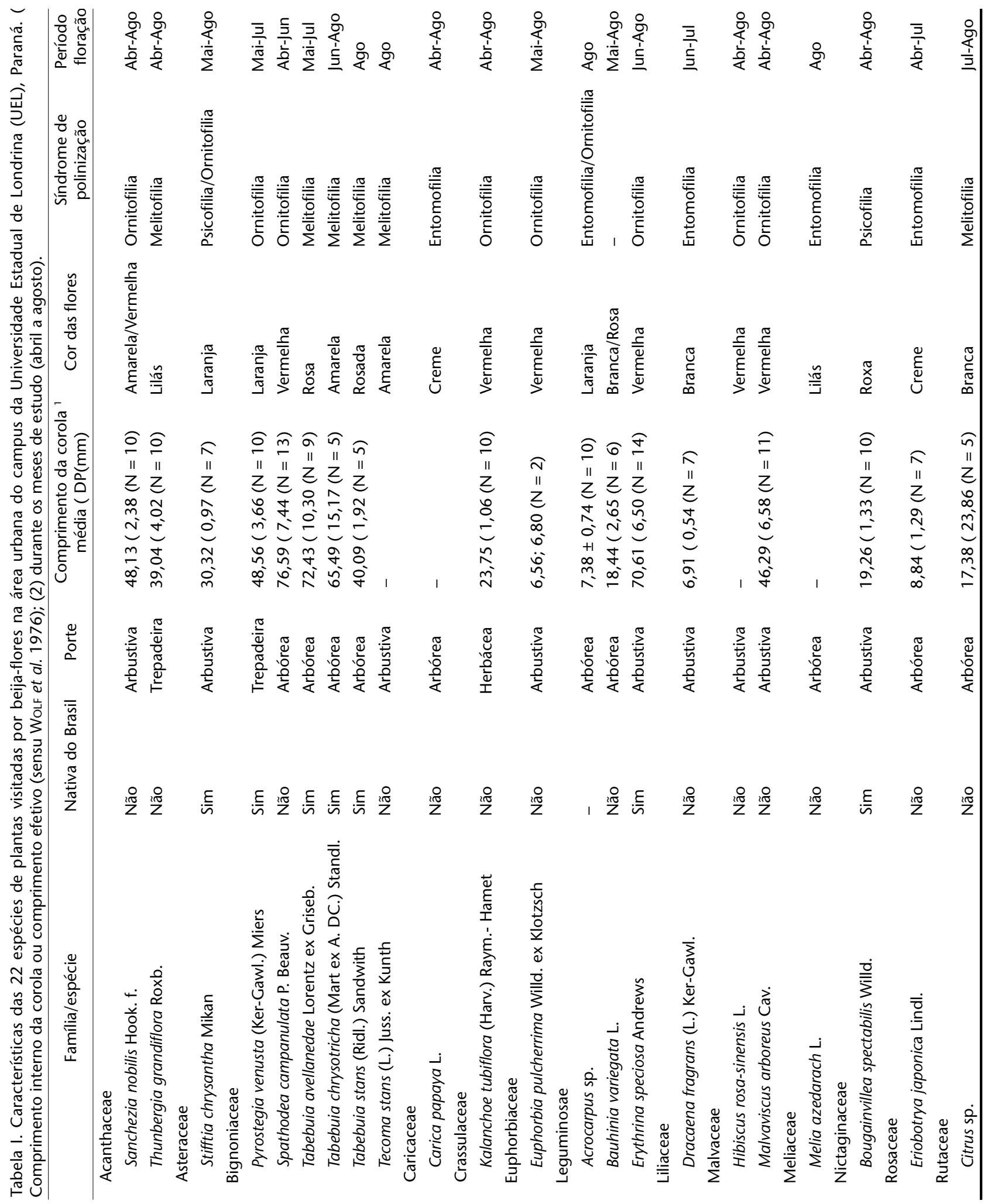




\section{Beija-flores}

Dez espécies de beija-flores foram observadas (Tab. II). Registrados durante todo o período de outono e inverno, Amazilia lactea (Lesson, 1832), Hylocharis chrysura (Shaw, 1812), Melanotrochilus fuscus (Vieillot 1817) e Chlorostilbon aureoventris (d'Orbigny \& Lafresnaye, 1838) foram as mais freqüentes (> 50,0\% dos dias amostrados, $\mathrm{N}=45)$. Eupetomena macroura (Gmelin, 1788) e Amazilia versicolor (Vieillot, 1818) tiveram as menores freqüências de ocorrência (4,4\% e 6,7\%, respectivamente).

\section{Morfologia e papéis comunitários}

Com base nas características morfológicas (massa, comprimento do bico, comprimento da asa e relação entre comprimento do bico e comprimento da asa; Tab. II), assembléia de plantas visitadas (Tab. III) e papéis comunitários desempenhados, as dez espécies de beija-flores foram classificadas em três grupos: 1) espécies de bicos curtos (<20 mm), massa corpórea pequena a média $(\leq 5 \mathrm{~g})$ e relação bico/asa intermediária $(0,32$ 0,37): Amazilia lactea, Amazilia versicolor, Chlorostilbon aureoventris e Hylocharis chrysura. Generalistas, estes beija-flores - com exceção de Amazilia versicolor, raramente observado - forragearam em uma variedade de espécies vegetais, com tamanho e forma de corola variados, incluindo diversas flores adaptadas à polinização por insetos. Com freqüência, atuaram como pilhadores de néctar (INOuYe 1980, 1983) nas flores de corolas longas (e.g. M. arboreus). Em agrupamentos de flores ou espécies profusamente floridas, exibiram comportamento territorial. Dentre estas espécies, Amazilia lactea foi a mais generalista, observada em 17 espécies de plantas, 58,8\% das quais nãoornitófilas. 2) espécies de bicos curtos a médios (21-23 $\mathrm{mm}$ ), massa corpórea elevada e relação bico/asa baixa $(<0,30$; Melanotrochilus fuscus e Eupetomenta macroura) ou intermediária (Anthracothorax nigricollis Vieillot, 1817 e Leucochloris albicollis Vieillot, 1818). Estes beija-flores, à semelhança das espécies citadas anteriormente, possuem comprimento de bico que lhes permite explorar uma variedade de recursos florais. M. fuscus e $E$. macroura apresentam características típicas de beija-flores territoriais (especialmente relação bico/asa), condizentes com o comportamento observado; ambas se mostraram bastante agressivas. A. nigricollis e L. albicollis tiveram baixa freqüência na UEL, não sendo possível avaliar em detalhe seu comportamento e papel comunitário. As duas apresentam características de espécies generalistas, embora $A$. nigricollis só tenha sido observado em duas espécies de plantas. Em algumas ocasiões, L. albicollis atuou como parasita de território, visitando um indivíduo de Erythrina speciosa Andrews defendido por M. fuscus enquanto este se ausentava brevemente. 3 ) espécies de bicos longos ( $>30$ $\mathrm{mm})$, massa corpórea média a elevada (> 6 g) e relação bico/asa superior a 0,50. Phaethornis pretrei (Lesson \& Delattre, 1839) e Heliomaster squamosus (Temminck, 1823) forragearam em linhade-captura e possuem características típicas de espécies que percorrem rondas alimentares de alta recompensa. Devido ao seu bico longo, $P$. pretrei visitou de modo legítimo flores com corolas longas, que não receberam visitas de outros beija-flores (Pyrostegia venusta (Ker-Gawl.) Miers) ou em que beija-flores de bicos curtos foram capazes de obter o néctar somente por perfurações na base da corola (Sanchezia nobilis). Esta espécie não apresentou comportamento territorial e foi geralmente afastada das flores pelas demais.

\section{Territorialidade}

A maioria das espécies de beija-flores exibiu comportamento territorial, que incluiu vocalizações, displays visuais e ataques agressivos. As interações agonísticas foram observadas principalmente nas plantas visitadas por número elevado de espécies de beija-flores (Tab. III), a saber: Spathodea campanulata (visitada por sete espécies), Erythrina speciosa (7), Stifftia chrysantha Mikan (7), Bauhinia variegata L. (6), Malvaviscus arboreus (5) e Tabebuia avellanedae Lorentz ex Griseb. (5). Além destas, Sanchezia nobilis, visitada com elevada freqüência por A. lactea foi defendida por esta espécie.

Um total de 63 interações foi registrado entre os beijaflores (71,4\% interespecíficas; Tab. IV). A posição das espécies na hierarquia de dominância (incluídas somente espécies com mais de cinco interações) esteve positivamente correlacionada com massa corporal ( $r$-Spearman $=0,84 ; \mathrm{p}<0,02 ; \mathrm{N}=7$ ) e negativamente correlacionada com o comprimento do bico em relação ao comprimento da asa $(\mathrm{r}=-0,89 ; \mathrm{p}<0,01 ; \mathrm{N}=7)$. $E$. macroura, M. fuscus e L. albicollis foram os beija-flores dominantes, expulsando outras espécies em 100\%, 88,2\% e 57,9\% dos encontros interespecíficos, respectivamente. Em contraste, $C$. aureoventris e $P$. pretrei foram afastados em todos os encontros interespecíficos.

\section{Largura e sobreposição de nicho}

Amazilia lactea apresentou o nicho trófico mais amplo $\left(\mathrm{H}^{\prime}=2,16\right)$, seguida por $H$. chrysura $(1,89)$ e C. aureoventris $(1,83)$. Entretanto, A. lactea concentrou suas visitas em cinco das 17 espécies visitadas, o que é refletido pela equitabilidade moderada de suas visitas $(J=0,76)$. As espécies de nicho mais estreito foram A. nigricollis $(0,38)$ e A. versicolor $(0,95)$. A largura do nicho esteve moderadamente correlacionada à freqüência de ocorrência das espécies ( $\mathrm{r}=0,79 ; \mathrm{p}<0,01 ; \mathrm{N}=10)$.

Elevados valores de sobreposição no uso dos recursos florais foram observados entre alguns pares de espécies (Tab. V), sendo E.macroura e M. fuscus, as espécies com maior sobreposição $(0,98)$. Valores elevados foram também observados entre C. aureoventris e L. albicollis $(0,89)$, M. fuscus e H. chrysura $(0,86)$ e H. chrysura e C. aureoventris $(0,82)$.

\section{DISCUSSÃO}

\section{Assembléia de plantas}

O número de espécies de plantas visitadas por beija-flores na UEL é similar ao registrado em capões no Pantanal (21 espécies; Araújo \& SAZIma 2003) e algumas áreas de Floresta Atlântica no sudeste do Brasil (15-25: SNOW \& TeixeIra 1982, SNOw \& SNOW 1986, SAzima et al. 1996; mas veja Buzato et al. 2000). Adicionalmente, outras duas espécies encontradas no campus são poten- 
Tabela II. Espécies de beija-flores registradas na UEL, suas características morfológicas, freqüência de ocorrência na área de estudo ( $\mathrm{N}=$ 45 dias de amostragem) e largura do nicho trófico.

\begin{tabular}{|c|c|c|c|c|c|c|c|}
\hline Subfamília/Espécie & Massa $^{1}(\mathrm{~g})$ & $\operatorname{Bico}^{1}(\mathrm{~mm})$ & $\mathrm{Asa}^{1}(\mathrm{~mm})$ & Bico/Asa ${ }^{2}$ & $\mathrm{FO}^{3}(\%)$ & $\mathrm{H}^{\prime}{ }^{4}$ & $\mathrm{~J}^{5}$ \\
\hline \multicolumn{8}{|l|}{ Phaethornithinae } \\
\hline Phaethornis pretrei & 7,0 & 35 & 61 & 0,57 & 22,2 & 1,67 & 0,93 \\
\hline \multicolumn{8}{|l|}{ Trochilinae } \\
\hline Eupetomena macroura & 9,0 & 21 & 78 & 0,27 & 4,4 & 1,04 & 0,95 \\
\hline Melanotrochilus fuscus & 9,0 & 21 & 85 & 0,25 & 62,2 & 1,46 & 0,75 \\
\hline Anthracothorax nigricollis & 7,0 & 22 & 65 & 0,34 & 15,5 & 0,38 & 0,55 \\
\hline Chlorostilbon aureoventris & 3,8 & 19 & 51 & 0,37 & 53,3 & 1,83 & 0,94 \\
\hline Hylocharis chrysura & 4,0 & 19 & 55 & 0,35 & 68,9 & 1,89 & 0,86 \\
\hline Leucochloris albicollis & 8,0 & 22 & 63 & 0,35 & 15,5 & 1,55 & 0,87 \\
\hline Amazilia versicolor & 4,0 & 18 & 52 & 0,35 & 6,7 & 0,95 & 0,86 \\
\hline Amazilia lactea & 5,0 & 18 & 57 & 0,32 & 82,2 & 2,16 & 0,76 \\
\hline Heliomaster squamosus & 6,5 & 31 & 61 & 0,51 & 11,1 & 1,01 & 0,92 \\
\hline
\end{tabular}

(1) Massa corporal,comprimento do bico e comprimento da asa - medidas obtidas em GranTSAU (1988); (2) relação entre o comprimento do bico e o comprimento da asa (SNow \& SNow 1980); (3) freqüência de ocorrência (FO), em porcentagem (N =45); (4) largura do nicho trófico $\left(H^{\prime}\right)$, calculado a partir do índice de Shannon (KREBS 1999); (5) equitabilidade (J) das visitas aos recursos florais (KREBS 1999).

Tabela III. Freqüência de visitas das dez espécies de beija-flores às 22 espécies de plantas observadas. 0 primeiro número se refere ao número de visitas (em dias) e o segundo a sua freqüência relativa.

\begin{tabular}{|c|c|c|c|c|c|c|c|c|c|c|}
\hline \multirow{2}{*}{ Família/Espécie de planta } & \multicolumn{10}{|c|}{ Espécie de beija-flor } \\
\hline & $\mathrm{Pp}$ & $\mathrm{Em}$ & $\mathrm{Mf}$ & An & $\mathrm{Ca}$ & $\mathrm{HC}$ & La & Av & $\mathrm{Al}$ & Hs \\
\hline \multicolumn{11}{|l|}{ Acanthaceae } \\
\hline Sanchezia nobilis & $3(0.23)$ & & & & & & & & $24(0,24)$ & \\
\hline Thunbergia grandiflora & $1(0,08)$ & & & & & & & & & \\
\hline \multicolumn{11}{|l|}{ Asteraceae } \\
\hline Stifftia chrysantha & $2(0,15)$ & $1(0,25)$ & $6(0,16)$ & & $6(0,15)$ & $7(0,14)$ & $2(0,09)$ & & $10(0,10)$ & \\
\hline \multicolumn{11}{|l|}{ Bignoniaceae } \\
\hline Pyrostegia venusta & $2(0,15)$ & & & & & & & & & \\
\hline Spathodea campanulata & & $1(0,25)$ & $11(0,29)$ & $7(0,88)$ & $5(0,12)$ & $15(0,29)$ & & & $1(0,01)$ & $2(0,33)$ \\
\hline Tabebuia avellanedae & & & $2(0,05)$ & $1(0,12)$ & & $4(0,08)$ & & & $1(0,01)$ & $3(0,50)$ \\
\hline Tabebuia chrysotricha & & & & & $4(0,10)$ & $1(0,02)$ & $1(0,04)$ & & $3(0,03)$ & \\
\hline Tabebuia roseo-alba & & & & & & $1(0,02)$ & & & & \\
\hline Tecoma stans & & & & & & & & & $2(0,02)$ & \\
\hline \multicolumn{11}{|l|}{ Caricaceae } \\
\hline Carica papaya & & & & & & & & $1(0,20)$ & $3(0,03)$ & \\
\hline \multicolumn{11}{|l|}{ Crassulaceae } \\
\hline Kalanchoe tubiflora & & & & & & & & & $1(0,01)$ & \\
\hline \multicolumn{11}{|l|}{ Euphorbiaceae } \\
\hline Euphorbia pulcherrima & & & $1(0,03)$ & & & & & & $1(0,01)$ & \\
\hline \multicolumn{11}{|l|}{ Leguminosae } \\
\hline Acrocarpus sp. & & & $1(0,03)$ & & & & $1(0,04)$ & & & \\
\hline Bauhinia variegata & & & $1(0,03)$ & & $10(0,24)$ & $6(0,12)$ & $6(0,27)$ & $1(0,20)$ & $18(0,18)$ & \\
\hline Erythrina speciosa & $4(0,31)$ & $2(0,50)$ & $16(0,42)$ & & $10(0,24)$ & $10(0,20)$ & $7(0,32)$ & & $7(0,07)$ & \\
\hline \multicolumn{11}{|l|}{ Liliaceae } \\
\hline Dracaena fragrans & & & & & & & & & $1(0,01)$ & \\
\hline
\end{tabular}


Tabela III. Cotinuação.

\begin{tabular}{|c|c|c|c|c|c|c|c|c|c|c|}
\hline \multirow{2}{*}{ Família/Espécie de planta } & \multicolumn{10}{|c|}{ Espécie de beija-flor } \\
\hline & $\mathrm{Pp}$ & $\mathrm{Em}$ & $M f$ & An & $\mathrm{Ca}$ & $\mathrm{HC}$ & $\mathrm{La}$ & Av & $\overline{A l}$ & $\mathrm{Hs}$ \\
\hline \multicolumn{11}{|l|}{ Malvaceae } \\
\hline Hibiscus rosa-cinensis & & & & & & & & & $1(0,01)$ & $1(0,17)$ \\
\hline Malvaviscus arboreus & & & & & $4(0,10)$ & $6(0,12)$ & $5(0,23)$ & $3(0,60)$ & $22(0,22)$ & \\
\hline \multicolumn{11}{|l|}{ Meliaceae } \\
\hline Melia azedarach & $1(0,08)$ & & & & $2(0,05)$ & & & & $1(0,01)$ & \\
\hline \multicolumn{11}{|l|}{ Nictaginaceae } \\
\hline Bougainvillea spectabilis & & & & & & & & & $3(0,03)$ & \\
\hline \multicolumn{11}{|l|}{ Rosaceae } \\
\hline Eriobotrya japonica & & & & & & $1(0,02)$ & & & & \\
\hline \multicolumn{11}{|l|}{ Rutaceae } \\
\hline Citrus sp. & & & & & & & & & $1(0,01)$ & \\
\hline Total $(\mathrm{N})$ & 13 & & 38 & 8 & 41 & 51 & 22 & 5 & 100 & 6 \\
\hline
\end{tabular}

Beija-flores: (Pp) Phaethornis pretrei, (Em) Eupetomena macroura, (Mf) Melanotrochilus fuscus, (An) Anthracothorax nigricollis, (Ca) Chlorostilbon aureoventris, (Hc) Hylocharis chrysura, (La) Leucochloris albicollis, (Av) Amazilia versicolor, (Al) Amazilia lactea e (Hs) Heliomaster squamosus.

Tabela IV. Matriz de interações agonísticas registradas entre os beija-flores durante as visitas às flores ( $\mathrm{N}=63$ interações). Para cada espécie foi efetuada a somatória dos eixos horizontal (número de vezes em que cada espécie atacou) e vertical (número de vezes em que cada espécie foi agredida).

\begin{tabular}{|c|c|c|c|c|c|c|c|c|c|c|c|c|}
\hline $\begin{array}{l}\text { Subordinada } \\
\text { Agressora }\end{array}$ & $\mathrm{Pp}$ & Em & $\mathrm{Mf}$ & An & $\mathrm{Ca}$ & $\mathrm{Hc}$ & La & Av & $\mathrm{Al}$ & $\mathrm{Hs}$ & $\Sigma^{1}$ & $\Sigma^{2}$ \\
\hline $\mathrm{Pp}$ & & & & & & & & & & & 0 & 0 \\
\hline $\mathrm{Em}$ & & & 2 & & & 3 & & & 1 & & 6 & 6 \\
\hline $\mathrm{Mf}$ & 1 & & 6 & & 1 & 4 & 7 & & 2 & & 15 & 21 \\
\hline An & & & & & 1 & & & & & & 1 & 1 \\
\hline $\mathrm{Ca}$ & & & & & 1 & & & & & & 0 & 1 \\
\hline $\mathrm{Hc}$ & & & & 1 & 2 & 1 & & & 1 & & 4 & 5 \\
\hline La & 4 & & & & 1 & 2 & 8 & & 4 & & 11 & 19 \\
\hline$A v$ & & & & & & & & & & & 0 & 0 \\
\hline $\mathrm{Al}$ & 2 & & & & 3 & & 1 & 1 & 2 & & 7 & 9 \\
\hline $\mathrm{Hs}$ & & & & & & & & & 1 & & 1 & 1 \\
\hline$\Sigma^{1}$ & 7 & 0 & 2 & 1 & 8 & 9 & 8 & 1 & 9 & 0 & 45 & - \\
\hline$\Sigma^{2}$ & 7 & 0 & 8 & 1 & 9 & 10 & 16 & 1 & 11 & 0 & - & 63 \\
\hline
\end{tabular}

$\Sigma^{1}$ Somatória das interações interespecíficas $(\mathrm{N}=45) ; \Sigma^{2}$ somatória de todas as interações $(\mathrm{N}=63)$. As espécies de beija-flores são denominadas como na tabela III.

cialmente visitadas pelos beija-flores na UEL: Pachystachys lutea Nees e Justicia brandegeana Wassh. \& Lor. B. Sm (Acanthaceae). Das 22 espécies de plantas visitadas, sete (32\%: S. campanulata, E. speciosa, S. chrysantha, B. variegata, M. arboreus, T. avellanedae e S. nobilis) parecem caracterizar os recursos mais importantes para a comunidade de beija-flores estudada, devido ao elevado número de espécies visitantes e/ou elevada freqüência de visitas por uma ou mais espécies de beija-flores.

A proporção de espécies não-ornitófilas visitadas pelos beija-flores na UEL pode ser considerada alta se comparada a diversos outros estudos (33,3-39,6\%; SNOw \& SNOW 1986, ARIZMENDI \& ORnelas 1990, Cotton 1998b, Vasconcelos \& Lombardi 2001). Araujo \& Sazima (2003) encontraram resultado semel hante e sugeriram que o el evado número de plantas não-ornitófilas visitadas pelos beija-flores poderia estar relacionado à escassez de espécies ornitófilas. Na UEL, outras possíveis explicações incluem: (1) a dominância das espécies ornitófilas por beija-flores territoriais, levando beija-flores de menor habilidade competitiva a adotar um padrão de forrageamento mais generalista; e (2) maior abundância de flores não-ornitófilas, as quais podem for- 
Tabela V. Valores de sobreposição do nicho trófico (índice de Pianka, em KREBs 1999) para as espécies de beija-flores registradas na UEL.

\begin{tabular}{|c|c|c|c|c|c|c|c|c|c|c|}
\hline & $\mathrm{Pp}$ & $\mathrm{Em}$ & Mf & $\mathrm{An}$ & $\mathrm{Ca}$ & $\mathrm{Hc}$ & $\mathrm{La}$ & $\mathrm{Av}$ & $\mathrm{Al}$ & $\mathrm{Hs}$ \\
\hline $\mathrm{Pp}$ & - & 0,69 & 0,63 & 0 & 0,53 & 0,42 & 0,50 & 0 & 0,52 & 0 \\
\hline $\mathrm{Em}$ & & - & 0,98 & 0,40 & 0,73 & 0,79 & 0,61 & 0 & 0,26 & 0,22 \\
\hline Mf & & & - & 0,54 & 0,74 & 0,86 & 0,59 & 0,01 & 0,25 & 0,36 \\
\hline An & & & & - & 0,29 & 0,71 & 0 & 0 & 0,03 & 0,64 \\
\hline $\mathrm{Ca}$ & & & & & - & 0,82 & 0,89 & 0,38 & 0,61 & 0,15 \\
\hline $\mathrm{Hc}$ & & & & & & - & 0,65 & 0,34 & 0,47 & 0,52 \\
\hline La & & & & & & & - & 0,59 & 0,68 & 0 \\
\hline Av & & & & & & & & - & 0,66 & 0 \\
\hline Al & & & & & & & & & - & 0,04 \\
\hline $\mathrm{Hs}$ & & & & & & & & & & - \\
\hline
\end{tabular}

As espécies de beija-flores são denominadas como na tabela III.

necer néctar com valor energético similar ao de espécies polinizadas por beija-flores (ArIZMEndi \& OrNelas 1990). É possível ainda que determinadas características das plantas visitadas pelos beija-flores na área urbana da UEL (e.g. elevada proporção de espécies não-ornitófilas, espécies exóticas e de plantas arbóreas) reflitam simplesmente as características gerais dos recursos florais disponíveis neste tipo de paisagem.

\section{Beija-flores}

A comunidade de beija-flores registrada na UEL abrangeu 10 (> 80\%) das 12 espécies mencionadas para a área urbana do município de Londrina (Wеstсотт 1980). Este número é pequeno se comparado ao encontrado em outras comunidades na Colômbia (15 espécies: Kraemer et al. 1993, 16: Cotton 1998b) e na Costa Rica (14: Feinsinger 1976, 1978). Por outro lado, se aproxima do registrado no Brasil em áreas de Floresta Atlântica (Snow \& Teixeira 1982, Snow \& Snow 1986, Sazima et al. 1996, BuzATo et al. 2000) ou campos rupestres (VASCONCELos \& Lombardi 2001) e é superior ao registrado em capões no Pantanal (Araújo \& SAzIma 2003). Esta riqueza moderada de espécies em uma área urbana pode ser devida a uma série de fatores, incluindo o aumento na densidade de flores em comparação a áreas florestais (e.g. Ruszczyk 1986) e à capacidade das espécies em explorar os recursos disponíveis.

Contudo, a comunidade de beija-flores da UEL é composta principalmente por espécies generalistas de ampla distribuição geográfica no Brasil, que podem ser encontradas em áreas abertas, capoeiras e borda da mata (SICK 1997). Na área urbanizada da UEL, nota-se, por exemplo, a ausência de Phaethornis eurynome (Lesson, 1832) e Thalurania glaucopis (Gmelin, 1788), duas espécies comumente observadas em fragmentos florestais na região de Londrina (Anjos et al. 1997, Gimenes \& Anjos 2000, Anjos 2002).

As espécies registradas apresentaram diferentes massas, comprimento do bico, comprimento da asa e relação entre comprimento do bico e comprimento da asa. Estas diferentes combinações de características morfológicas reconhecidamente importantes na ecologia dos beija-flores (FEINSINGER \& COLWELl 1978, SNow \& SNOW 1980, Brown \& Bowers 1985), foram refletidas no conjunto de flores exploradas e na estratégia de forrageamento adotada. Os beija-flores se encaixaram bem nos papéis comunitários propostos por FeINSINGER \& COLWEll (1978). A relação entre comprimento do bico e comprimento da asa, sugerida por $S_{\text {NOW }} \& S_{\text {NOW }}(1980)$ como um bom indicador da estratégia de forrageamento adotada por beija-flores, também se mostrou adequada; pôde ser ainda utilizada para indicar a posição de uma dada espécie na hierarquia de dominância.

A maioria das espécies de beija-flores exibiu comportamento territorial, com destaque para $M$. fuscus que, por apresentar elevada freqüência e habilidade competitiva, pode ser considerada uma espécie organizadora (FEINsinger 1978, ARIZMENDI \& ORNelas 1990) na comunidade de beija-flores da UEL. Apenas E. macroura, ocasionalmente registrada, afastou M. fuscus das flores. Uma importante influência no custo da defesa de um território é o modo como este é defendido, o qual é por sua vez influenciado pela posição de uma espécie ou indivíduo na hierarquia de dominância (Wolf \& HaInsworTh 1971). M. fuscus pode facilmente afastar outras espécies de seus recursos florais. Por outro lado, para um beija-flor como E. macroura, ocasional na área de estudo, de elevada demanda energética (McMillen \& Carpenter 1977, Brown et al. 1978) e com um número elevado de competidores, defender os recursos florais disponíveis no local pode não ser vantajoso (STILEs \& Wolf 1970).

A massa corporal é um importante fator nos sistemas de dominância envolvendo beija-flores (StILES \& Wolf 1970, ArIzmendi \& Ornelas 1990, Kraemer et al. 1993). Analisando-se o nível de agressividade em relação à massa corporal dos beijaflores, se observa claramente que as espécies maiores foram 
dominantes sobre as menores na área de estudo, restringindo seu acesso às flores defendidas. Por outro lado, uma vez que as espécies de menor massa têm menores demandas energéticas totais (McMillen \& Carpenter 1977, Brown et al. 1978), estas foram capazes de explorar uma variedade de flores alternativas, ignoradas ou visitadas com baixa freqüência pelas espécies dominantes.

Em termos gerais, as espécies com nichos tróficos mais amplos, foram também as mais freqüentes, o que pode indicar que o ambiente urbano seria mais favorável às espécies mais generalistas. Ao mesmo tempo, os resultados sugerem que, para espécies com freqüência de ocorrência muito baixas, as medidas de largura do nicho trófico deveriam ser interpretadas com cautela, como no caso de A. nigricollis, E. macroura e A. versicolor, cuja morfologia lhes permite explorar uma variedade de recursos maior que a observada no presente estudo. Os elevados valores de sobreposição no uso dos recursos florais observados para a comunidade de beija-flores na UEL podem ter decorrido do fato de seis espécies de plantas receberem visitas freqüentes de pelo menos metade das espécies de beija-flores presentes no local.

Alguns pares de espécies, com destaque para M. fuscus e E. macroura, além de apresentarem grande sobreposição no uso das flores, possuem morfologia e comportamento semelhantes, sugerindo potencial competição entre elas. Interações agressivas interespecíficas foram, de fato, observadas entre os beijaflores, especialmente nas plantas visitadas por um maior número de espécies. Contudo, a interpretação das medidas de sobreposição do nicho em relação à competição interespecífica deve ser feita com cautela e levando em consideração a abundância dos recursos (Pité \& Avelar 1996, Latham 1999). Estas medidas podem não revelar o grau de exclusão de plantas potencialmente utilizadas ou a intensidade da competição entre os beija-flores (FEINSINGER 1978), uma vez que um determinado par de espécies pode explorar um mesmo conjunto de flores de modo espacialmente ou temporalmente distinto.

\section{Papel na polinização}

Observações mais detalhadas da biologia floral e ecologia da polinização das espécies visitadas na UEL são necessárias para avaliar o papel dos beija-flores na polinização destas. Entretanto, em algumas situações a atuação dos beija-flores como polinizadores pode ser inferido com base no comportamento de visita, nas características das flores e em informações obtidas na literatura. Algumas das espécies encontradas na UEL e conhecidamente polinizadas por beija-flores no Brasil são Pyrostegia venusta (GobatTo-Rodrigues \& STORT 1992) e Erythrina speciosa (Almeida \& Alves 2003). Outras, potencialmente polinizadas, incluem Stifftia chrysantha, visitada de modo legítimo pela maioria dos beija-flores observados e Sanchezia nobilis, visitada legitimamente por $P$. pretrei. Por outro lado, em diversas espécies de corolas longas (Malvaviscus arboreus, Tabebuia avellanedae, T. chrysotricha, Tecoma stans, Sanchezia nobilis), os beija-flores de bicos curtos com freqüência obtiveram o néctar por perfurações na base das corolas, atuando como pilhadores.
A coevolução difusa é considerada em muitos casos um importante fator na organização das comunidades de beijaflores (SNow \& SnOw 1980, CotTon 1998a, Buzato et al. 2000). No entanto, na área urbana da UEL e provavelmente em outras áreas de condições ambientais similares, as interações observadas entre a assembléia de plantas e a comunidade de beija-flores parece ser mais um resultado das circunstâncias do que de processos coevolutivos. Paisagens urbanas parecem ser ocupadas principalmente por espécies com menores exigências ecológicas em relação ao alimento e capazes de se adaptarem a ambientes abertos. Ao contrário, espécies consideradas mais especializadas ou associadas a ambientes florestais podem sofrer redução tanto em número de espécies quanto em sua abundância nestas áreas, uma vez que a simples disponibilidade de alimento na cidade não garantiria sua sobrevivência e permanência em áreas urbanas.

\section{AGRADECIMENTOS}

Ao herbário da Universidade Estadual de Londrina pela identificação das plantas. À Ana Odete S. Vieira, Silvia Helena Sofia, Graziele Hernandes Volpato, José Fernando Pacheco e um revisor anônimo pelas sugestões ao manuscrito. L. dos Anjos agradece pela bolsa de Produtividade em Pesquisa concedida pelo CNPq (350054/95-9).

\section{REFERÊNCIAS BIBLIOGRÁFICAS}

Almeida, E.M. \& M.A.S. Alves. 2003. Comportamento de aves nectarívoras em Erythrina speciosa Andrews (LeguminosaePapilionoideae) em uma área de Floresta Atlântica, Ilha Grande, Rio de Janeiro. Revista de Etologia, São Paulo, 5 (1): 1521.

Anjos, L. 2002. Forest bird communities in the Tibagi river hydrographic basin, southern Brazil. Ecotropica, Bonn, 8: 67-79.

Anjos, L.; K.L. Schuchmann \& R. Berndt. 1997. Avifaunal composition, species richness, and status in the Tibagi river basin, Parana State, Southern Brazil. Ornitologia Neotropical, Montreal, 8: 145-173.

AraúJo, A.C \& M. Sazima. 2003. The assemblage of flowers visited by hummingbirds in the "capões" of southern Pantanal, Mato Grosso do Sul, Brazil. Flora, Jena, 198: 1-9.

Arizmendi, M.C. \& J.F. Ornelas. 1990. Hummingbirds and their flower resources in a tropical dry forest in Mexico. Biotropica, St. Louis, 22 (2): 172-180.

Brown, J.H. \& A. Kodric-Brown. 1979. Convergence, competition, and mimicry in a temperate community of hummingbirdpollinated flowers. Ecology, Washington, 60 (5): 1022-1035.

Brown, J.H.; W.A. CAlder \& A. Kodrick-Brown. 1978. Correlates and consequences of body size in nectar-feeding birds. American Zoologist, Lawrence, 18: 687-700.

Brown, J.H. \& M.A. Bowers. 1985. Community organization in hummingbirds: relationships between morphology and ecology. Auk, Lawrence, 102 (2): 251-269. 
Buzato, S.; M. Sazima \& I. SAzima. 2000. Hummingbird-pollinated floras at three Atlantic Forest sites. Biotropica, Lawrence, 32 (4b): 824-841.

CotTon, P.A. 1998a. Coevolution in an Amazonian hummingbird-plant community. Ibis, London, 140: 639-646.

Cоттоn, P.A. 1998b. The community organization of a lowland Amazonian forest. Ibis, London, 140: 512-521.

FAEgri, K. \& L. VAN DER PIJL. 1979. The principles of pollination ecology. Oxford, Pergamon Press, 248p.

Feinsinger, P. 1976. Organization of a tropical guild of nectarivorous birds. Ecological Monographs, Washington, 46 (3): 257-291.

FeINSINGER, P. 1978. Ecological interactions between plants and hummingbirds in a tropical community. Ecological Monographs, Washington, 48 (3): 269-287.

Feinsinger, P. \& R.K. Colwell. 1978. Community organization among Neotropical nectar-feeding birds. American Zoologist, Lawrence, 18: 779-795.

Gimenes, M.R. \& L. Anjos. 2000. Distribuição da avifauna em um fragmento florestal do campus da Universidade Estadual de Londrina, Norte do Paraná, Brasil. Revista Brasileira de Zoologia, Curitiba, 17 (1): 263-271.

Gobatto-Rodrigues, A.A. \& M.N.S. Stort. 1992. Biologia floral e reprodução de Pyrostegia venusta (Ker-Gawl) Miers (Bignonieaceae). Revista Brasileira de Botânica, São Paulo, 15 (1): 37-41.

Grantsau, R. 1988. Os beija-flores do Brasil: uma chave de identificação para todas as formas de beija-flores do Brasil. Rio de Janeiro, Ed. Expressão e Cultura, 233p.

InOuYE, D.W. 1980. The terminology of floral larceny. Ecology, Washington, 61: 1251-1253.

INOUYE, D.W. 1983. The ecology of nectar robbing, p. 153-174. In: B. Bentley \& T. Elias (Eds). The biology of nectaries. New York, Columbia University Press, 259p.

Kraemer, M.; U. Schmitt \& K.L. Schuchmann. 1993. Notes on the organization of a Neotropical high-altitude hummingbirdflower community. p. 61-65. In: W. BARTHLOTT; C.M. NAUMANN; L. Schimidt-Loske \& K.L. Schuchmann (Eds). Animal-plant interactions in tropical environments. Bonn, Results of the Annual Meeting of the German Society for Tropical Ecology, 227p.

Krebs, C. J. 1999. Ecological methodology. Menlo Park, Addison-Wesley Educational Publishers, 620p.

LATHAM, J. 1999. Interespecific interactions of ungulates in European forests: an overview. Forest Ecology and Management, Amstedam, 120: 13-21.

McMillen, R.E. \& F.L. Carpenter. 1977. Daily energy costs and body weight in nectarivorous birds. Comparative Biochemistry and Physiology, Oxford, 56A (3): 439-441.
MendonçA, L.B. \& L. Anjos. 2003. Bird-flower interactions in Brazil: a review. Ararajuba, Seropédica, 11 (2): 195-205.

Missouri Botanical Garden. 2004. Disponível on-line em www.mobot.org/W3T/Search/vast.html

Pité, M.T. \& T. Avelar. 1996. Ecologia das populações e das comunidades. Lisboa, Fundação Calouste Gulbenkian. 311p.

Ruszczyk, A. 1986. Hábitos alimentares de borboletas adultas e sua adaptabilidade no ambiente urbano. Revista Brasileira de Biologia, Rio de Janeiro, 46 (2): 419-427.

SAZIMA, M.; S. BuZATo \& I. SAZIMA. 1996. An assemblage of hummingbird-pollinated flowers in a montane forest in southeastern Brazil. Botanica Acta, New York, 109: 149-160.

SICK, H. 1997. Ornitologia brasileira. Rio de Janeiro, Ed. Nova Fronteira, 912p.

SnOw, D.W. \& B.K. SnOw. 1980. Relationships between hummingbirds and flowers in the Andes of Colombia. Bulletin of the British Museum (Natural History: Zoology), London, 38 (2): 105-139.

SNOw, D.W. \& B.K. SNow. 1986. Feeding ecology of hummingbirds in the Serra do Mar, southeastern Brazil. El Hornero, Buenos Aires, 12: 286-296.

Snow, D.W. \& D.L. Teixeira. 1982. Hummingbirds and their flowers in the coastal mountains of southeastern Brazil. Journal für Ornithology, Frankfurt, 123 (4): 446-450.

Stiles, F.G. \& L.L. Wolf. 1970. Hummingbird territoriality at a tropical flowering tree. Auk, Lawrence, 87 (3): 467-491.

StiLes, F.G. 1978. Temporal organization of flowering among the hummingbird foodplants of a tropical wet forest. Biotropica, Lawrence, 10 (3): 194-210.

Stiles, F.G. 1981. Geographical aspects of bird-flower coevolution, with particular reference to Central America. Annals of the Missouri Botanical Garden, St. Louis, 68: 323-351.

VASCONCELOS, M.F. \& J.A. LOMBARDI. 2000. Espécies vegetais visitadas por beija-flores no meio do verão no Parque Estadual da Pedra Azul, Espírito Santo. Melopsittacus, Belo Horizonte, 3 (1): 36-41.

Vasconcelos, M.F. \& J.A. Lombardi. 2001. Hummingbirds and their flowers in the campos rupestres of southern Espinhaço Range, Brazil. Melopsittacus, Belo Horizonte, 4 (1): 3-30.

WestCotT, P.W. 1980. Descrição das aves encontradas na área urbana de Londrina - Paraná, primeira parte: aves não passeriformes. Semina, Londrina, 6 (2): 59-66.

Wolf, L.L. \& F.R Hainsworth. 1971. Time and energy budgets of territorial hummingbirds. Ecology, Washington, 52: 980988.

Wolf, L.L.; F.G. Stiles \& F.R. Hainsworth. 1976. Ecological organization of a tropical highland hummingbird community. Journal of Animal Ecology, Oxford, 32: 349-379.

Recebido em 01.VII.2004; aceito em 05.I.2005. 